\title{
De apelativos y calificadores en español
}

\author{
Emma Martinell Gifre y Cristina Illamola i Gómez (Universitat de \\ Barcelona, España)
}

\begin{abstract}
In this article, we will deal with forms whose function within the discourse is basically to qualify (in general, they are essentially attributes) and whose proliferation responds to a need that goes far beyond linguistic issues, since a group of social factors must be taken into consideration. Thus, we will examine pieces that can be defined as qualifying adjectives (cateto/cateta, cenizo/ceniza), as derived nouns (bocazas), as nouns bearing a metaphorical value (cobaya, tesoro), as nominalized adjectives (rubiales. frescales), as well as those that respond to an appealing function (tronco, enano) or others that come from cultural traditions (adán, ídolo, judas).

After, we present our starting hypotheses: 1) the ending - $a$, as a carrier of reference to 'woman', is diluted in the heterogeneity of considered forms; 2) the role of the article (el/la, un/una) is decisive in the presence of these forms; 3 ) newly formed forms coexist with old forms; and, above all, 4) in the use of these appreciative forms there does not seem to be awareness of a woman/man reference emanating from the end in $-a$ or $-o$, or any other.

To corroborate them, we have selected around forty voices and examined their use and prevalence in the online versions of CORDE, CREA, CORPES XXI, various editions of Diccionario de la lengua (RAE) and Diccionario inverso de la lengua española.

Finally, the analysis leads us to affirm that, in the use of these nominal forms, the endings in $-e,-i /-i s$ or $-o$ to apply to a female referent are not rejected, nor, on the contrary: many forms ending in $-a$ do not know a transformation into $-o$ to apply to a male referent. Moreover, the language avoids any possible ambiguity with the application of the article, derivation by suffixes or by an attributive structure.
\end{abstract}

Keywords: appellatives, qualifiers, Spanish gender, grammatical gender, variation

En la tesis doctoral de Christel Larsson, que trataba de la variación en el nombramiento de las mujeres profesionales en España, y en sus otras investigaciones (2008, 2014), se mostraba la creciente tendencia a aplicar la desinencia -a de género gramatical femenino a muchos nombres profesionales (fiscala, médica, jueza), nombres de profesiones históricamente propias de hombres, pero que progresivamente van siendo desempeñadas por mujeres. La autora concluía, entonces, que dicha creación neológica por derivación se da tanto cuando el 
nombre profesional termina en -o (médico, bombero), como cuando el nombre termina en consonante (pescador, capataz, cónsul).

En esta ocasión, hemos querido reflexionar también sobre parejas de voces como el modisto/la modista, o en parejas como el/la atleta, el/la terapeuta (del griego); en palabras invariables, como el adjetivo sustantivado el/la contable; en formas invariables, como el/la guardaespaldas. Todas son denominaciones de actividades profesionales en las que no se tiende a cambiar la terminación para la referencia genérica del referente.

Todas esas reflexiones nos han llevado -pues habíamos decidido avanzar en el conocimiento y profundización de esta pequeña parcela de la lengua (Bosque 1999; Demonte 1999; Real Academia Española 2010; García, Aliaga y Lázaro Mora 2003; Cabré et al. 2000; Serrano-Dolader 2010; M. C. Junyent 2013 a y b; Lledó Cunill 2011; Bosque 2012)- a ir más allá del terreno de las denominaciones de profesiones, o cargos, pues en ese campo intervienen factores sociológicos y culturales de peso (Gómez Torrego 2011; Hellinger y Bussmann 2002).

Más concretamente, nos formulamos la siguiente pregunta como punto de partida: ¿es cierto que se acepta la asociación estable de la -a de femenino con un referente de género/sexo ${ }^{1}$ femenino, y que, paralelamente, la -o de masculino alude, para la colectividad hablante, a un referente de género/sexo masculino? Puede que sea así y es nuestro cometido aquí analizar u exponer esta asociación a través de la creatividad léxica.

Pero hay mucho más, y es precisamente esa zona en la que centramos nuestra investigación. Por un lado, es un campo que compete a la morfología derivativa o léxica, a la neología, a la sintaxis, a la semántica, y a la pragmática. Como marco, está la psicología, el sentimiento de los hablantes. Y como aplicación, la enseñanza del español, lengua románica, en la que hay rasgos gramaticales decisivos y característicos, como la determinación mediante el artículo, la derivación por sufijos, o la estructura atributiva. Por lo tanto, ahondaremos más allá de la alternancia -o/-a.

Concretamente, estamos en la esfera de los nombres apelativos que califican, que describen. La gramática viene distinguiendo entre «adjetivos calificativos especificativos» y «explicativos» (Alcina y Blecua 1975; Llorach 1978; Bello 1887; Fernández Ramírez 1985; Real Academia Española 1973, 2010; Bosque y Demonte 1999), y se reconoce una categoría nominal, a la que pertenecen sustantivos y adjetivos, con formas prototípicas que se sitúan en el extremo de ese contínuum nominal, pero con formas con usos, no necesariamente estables, intermedios (procesos de sustantivación y de adjetivación) (Briz 1990).

Con todo, hay muchos tipos de formas para las que la reflexión expuesta entre los finales -o/-a no resulta pertinente: primero, el español ha heredado formas invariables en -a, procedentes de neutros griegos (demócrata, hipócrita, psicópata, patriarca/matriarca), que alternan el uso como adjetivos con el uso como sustantivos. Ciertamente una dificultad para los que aprenden español, que optan por atribuir artículo femenino a esas formas (de origen clásico) terminadas en -a: *la mapa, la problema, por ejemplo. En esta última función, es el

\footnotetext{
${ }^{1}$ No pretendemos en este artículo ahondar en cuestiones de sexo/género. Cfr. C. Junyent 2013 a y b; Manrique Sabogal 2012, entre muchos otros.
} 
artículo el que identifica el referente (el/la ácrata), en una operación similar a la que se lleva a cabo en las parejas el/la testigo, el/la reo ( ¿rea ${ }^{2}$ ). Son los llamados «sustantivos comunes» en cuanto al género. Además, existen los llamados «nombres epicenos», como personaje o víctima, que se combinan solo con un artículo: el personaje (ella es el personaje protagonista; él fue la víctima inocente).

Segundo, el español cuenta con adjetivos calificadores invariables, de una sola terminación, como capaz, hábil, cortés o cobarde. En su uso adjetival, van acompañados de un sustantivo; en su función sustantivada, en cambio, podemos decir que es el contexto sintáctico o situacional el que favorece la interpretación del género masculino o femenino. No creemos, ni consta en los corpus, que nunca se hayan feminizado.

Tercero, el español cuenta con adjetivos calificadores terminados en cualquiera de las dos desinencias (cateto/cateta, enano/enana -aplicado a los hijos-, lo mismo que petardo/petarda, cenizo/ceniza -donde la coincidencia de la forma en -a con otra entidad dificulta el avance de su uso como calificador).

Para esta ocasión hemos seleccionado un total de 112 formas (véanse las Tablas 4 y 5 más adelante) limitándonos al español europeo, pues dejamos para una futura aproximación la variabilidad del español americano, así como la comparación con las formas peninsulares. Así pues, hemos tomado en consideración formas calificadoras, de función apelativa (Bañón 1993; González Rodríguez 1979; García Dini 1998; Gómez Montoya 2009), fuera del campo de las denominaciones de la actividad profesional.

Tenemos una hipótesis de partida: la exclusividad de la desinencia -a, como portadora prototípica del contenido de género femenino (y, por extensión, dado que trabajamos con formas alusivas a rasgos atribuibles a referentes marcados no solo por el rasgo +animado, sino también con el rasgo +humano, al género 'mujer') queda diluida, sobre todo, por la heterogeneidad de las formas en cuestión. Dicho de otro modo, hay muchísima riqueza en la vocal o consonante finales de una forma, principalmente por la abundancia de sufijos (un modelo productivo de neología léxica). A esta riqueza se le suma la adquisición de valores metafóricos, fenómeno que se da tanto en adjetivos sustantivados como en sustantivos de modo permanente.

Hay, en español (y en otras lenguas), nombres propios que han adquirido valor y función de nombre común. Se trata de una traslación por metonimia del referente a sus propiedades más representativas. El segundo paso es la aplicación de ese contenido descriptivo a otro referente (+humano), poseedor de ese rasgo a ojos de quien habla. Gracias a la referencia histórico/cultural (adán, tarzán, mesalina) se aplicarán -sea cual sea su final- a referente mujer u hombre.

\footnotetext{
${ }^{2}$ La forma testiga es conocida, sobre todo, por el uso que hizo de ella la actriz Chus Lampreave en una película de Almodóvar, y a partir de la cual se popularizó, al decir: «soy testiga de Jehová». Aunque se documenta algún caso de la forma femenina rea, son pocos los casos y parece más frecuente en el español americano: «Permiten a rea amamantar a hijo» («Judicial: esta sindicada por drogas». Crítica. Panamá: Editora Panamá, S. A., 2004-11-19, CORPES XXI, consulta realizada el 12/12/2017).
} 
[...] es sin duda el lugar idóneo de ligue para un tarzán como Álvarez Álvarez. Figuero, Javier, 1981, UCD: La "empresa" que creó Adolfo Suárez. Historia, sociología y familias del suarismo. Barcelona: Grijalbo. [CREA, consultado el 25/07/2017].

No necesariamente son formas muy antiguas, tal es el caso de maruja, que ya ha dado lugar a formas derivadas: marujeo, marujear ${ }^{3}$.

El español conoce la aplicación metafórica de nombres de animales, pues algo de su aspecto o de su comportamiento favorece que haya una traslación de rasgos, en un proceso de connotación. Se aplican a seres sexuados con total independencia de cómo sea el final de la forma (cobaya, hormiguita, lince, merluzo, tiburón). El final de la palabra para nada interfiere en su aplicación, ni 'incita' a su modificación.

Mientras tanto, ha picado como un merluzo o un jurel, como si fuera meramente un mero, en las procelosas aguas del caladero marroquí y aún lleva el anzuelo entre los dientes. La Razón, 20/12/2001: Morocotudo. [CREA consultado el 25/07/2017].

Muy alejadas de estas formas están los préstamos ya asimilados y con forma españolizada (líder, crack/crac, sumiller), cuyo final no remite a nada, no evoca nada que guarde relación con el morfema de género ${ }^{4}$.

Samantha Vallejo-Nágera no es solo una recién estrenada y tía feliz porque Colate y Paulina Rubio ya son padres. Es una chef solidaria que os necesita los días 18 y 19. [CORPES XXI consultado el 24/07/2017].

En cuanto a la aplicación metafórica de sustantivos comunes (Llamas Saíz 2005; Chamizo 2005), fenómeno de productividad incesante, vemos cómo engorro o plomo terminan en -o, que pelota, leyenda o estrella terminan en -a, y que otros sustantivos terminan en otra vocal o en consonante (ángel, pilar). Lo que ocurre con estas formas es que, de forma contraria a lo que veíamos líneas más arriba, no es el artículo, masculino o femenino, el que identifica el género/sexo del referente humano aludido o calificado, porque la estructura (atributiva) es siempre la misma: ser (el) pilar (de), ser (un) diamante (en bruto), ser (una) leyenda (viva), etc. Este es el un/una llamado «enfático» (Fernández Ramírez, 1985), que favorece una interpretación evaluativa y metafórica del adjetivo atributo, con la suspensión de la concordancia de género y de número. Por lo tanto, solo en el contexto se localiza(n) la(s) marca(s) de identificación (mi abuela materna es el pilar de la familia, este recogepelotas es un diamante en bruto, Usain Bolt se ha convertido en una leyenda viva, con el paso de los años llegará a ser una vieja gloria). La estructura es atributiva (con ser, no con estar, pero sí con algunos verbos más de los llamados «de cambio»). Estará de acuerdo el lector en que ni el hablante ni sus oyentes/lectores echan en falta modificaciones que, en estos casos, habrían de recaer en los artículos.

Hay también aplicación metafórica en otro tipo de nombres calificadores, palabras compuestas, que abarcan, aparte de numerosos nombres profesionales expresados con un tono

\footnotetext{
${ }^{3}$ Quizás la aparición documentada de marujo/marujón sería un indicio del cierre de este proceso.

${ }^{4}$ En el siguiente enlace de la Fundéu (https://www.fundeu.es/recomendacion/lider-lideresa-femenino/) se explica la aceptación del femenino lideresa, y su éxito sobre todo en América.
} 
cuando menos irónico (picapleitos, matasanos, pintamonas, destripaterrones) (Timofeeva 2005) y calificadores (cantamañanas, correveidile, metomentodo, sabelotodo, mandamás). Una vez más será el artículo previo -el indeterminado en estos casos-, el que arrojará luz sobre el género/sexo del ser humano aludido.

Hemos mencionado maruja como formación moderna, igual que se podría mencionar gorila, versión popular y coloquial de guardaespaldas. Pero marmota, piraña, тиеrmo, babosa, roña, roñoso/-a o roñica, ¿son palabras antiguas? Mejor dicho, ¿su aplicación como apelativo, como designador y caracterizador de persona es antigua o moderna? La explicación para el significado de muchas formas remite al pasado (sablista, derivado de sable; en cotilla, derivada de cota), pero ya no queda nada de la referencia inicial en su aplicación actual.

Tenemos que referirnos a «lo coloquial», en parte para justificar que hasta ahora no hayamos consultado el corpus Val.Es.Co 2.0, en el apartado de búsqueda morfológica avanzada, ni el corpus Cola, de la Universidad de Bergen, ni tampoco el Diccionario del español coloquial que mantiene en la red Victoriano Gaviño ${ }^{5}$. Es debido a una postura personal sobre el alcance del término coloquial $^{6}$. Reconocemos, con todo, que el peso de los medios de comunicación, sobre todo de la televisión (siempre hablamos de España) y del auge de la comunicación establecida en las redes sociales, es una fuente de creación léxica (o semántica) hoy en día, y, en gran parte, en manos o en boca de los jóvenes (Briz Gómez 2001; 2010; Beinhauer 1978; Vigara Tauste 1992; Moral 2003).

El lenguaje de los jóvenes constituye una modalidad lingüística, una fuerza generadora de cambios (Rodríguez González 2002; Rodríguez González 2002; Zimmermann 2002; Hernández Alonso 1991; Marimón-Llorca y Santamaría-Pérez 2001). A la vez, lo que contenga el vocabulario juvenil de creación léxica y de recreación semántica, creemos que no aporta datos diferentes de los que han proporcionado las fuentes a las que hemos acudido. Antonio Briz y otros miembros del grupo Val.Es.Co, así como las múltiples entrevistas semidirigidas recopiladas por el proyecto PRESEEA $^{7}$ en diversas ciudades hispanohablantes, se basan en un corpus de conversaciones reales grabadas. Para ellos, en el registro oral, la planificación sobre la marcha supone un menor control sobre la intervención/el texto. Reconocen, claro está, lo coloquial como prototípico de la periferia de lo coloquial y, asimismo, que hay una coloquialidad directa y otra referida, imitada, recreada. Esa visión más amplia conviene mejor a nuestra investigación.

Para este estudio aproximativo (entre cuyos objetivos reside también el reseguir la pervivencia o la modernidad de alguna de estas formas), nos hemos valido de los corpus que la RAE pone a disposición del especialista: el CORDE, el CREA y el CORPES XXI. Si bien somos conscientes de sus limitaciones, principalmente por la distribución de las fuentes entre

\footnotetext{
5 Los citados corpus y el diccionario están disponibles en http://www.valesco.es/?q=es/corpus, en http://www.colam.org/om_prosj-espannol.html y en http://www.coloquial.es/es/diccionario-del-espanolcoloquial/, respectivamente.

${ }^{6}$ En su tesis doctoral (1973), E. Martinell defendía que lo coloquial pertenecía al coloquio, al diálogo, fuera este real o ficticio. Para la autora, lo coloquial no implica necesariamente un registro popular, ni vulgar, ni remite a una lengua sectorial, la de los jóvenes, por ejemplo (objeto del proyecto Cola y del Grupo Val.Es.Co).

${ }^{7} \mathrm{Cfr}$. http://preseea.linguas.net/Inicio.aspx
} 
España y América $^{8}$, consideramos que para el objetivo que perseguimos ahora son plenamente útiles. Así, mediante la búsqueda en los tres corpus, siempre en orden cronológico, hemos podido constatar la antigüedad de ciertas voces. Véanse las siguientes tablas a modo de ejemplo, donde clasificamos por corpus y según el género/sexo al que se refiere el sustantivo (bien mediante artículo, bien por el cotexto).

\begin{tabular}{lccc}
\multicolumn{1}{c}{ Fuente } & Masculino & Femenino & Total \\
\hline CORDE & 1 & 0 & 1 \\
\hline CREA & 24 & 0 & 24 \\
\hline CORPES XXI & 38 & 0 & 38 \\
\hline
\end{tabular}

Tabla 1: Ocurrencias para pinchadiscos en los distintos corpus

\begin{tabular}{|lccc|}
\hline \multicolumn{1}{|c}{ Corpus } & Masculino & Femenino & Total \\
\hline CORDE & 0 & 0 & 0 \\
\hline CREA & 24 & 0 & 24 \\
\hline CORPES XXI & 216 & 14 & 230 \\
\hline
\end{tabular}

Tabla 2: Ocurrencias para $D J^{9}$ en los distintos corpus

\begin{tabular}{|cccc|}
\multicolumn{1}{c}{ Fuente } & Masculino & Femenino & Total \\
\hline CORDE & 18 & 2 & 20 \\
\hline CREA & 46 & 2 & 48 \\
\hline CORPES XXI & 28 & 0 & 28 \\
\hline
\end{tabular}

Tabla 3: Ocurrencias para pasmarote en los distintos corpus

También es cierto que algunas se documentan ya desde antiguo, pero son otros los valores recogidos, todavía no metaforizados, como ocurre, entre otros, con jeta, manitas, lumbrera o trepa. Por último, de entre todas las que hemos seleccionado, otras, por su reciente creación, como mensaca o choni, no se documentan en el CREA, pero sí en CORPES XXI.

"[...] juega al glamour del universo choni: "Yo domingueo", se lee en lentejuelas en una camiseta mientras las modelos pasean barras de pan [...]”. Fernández-Santos, Elsa: «La confusión corona Cibeles». El País.com. Madrid: elpais.com, 2009-02-25.

"[...] Fue El Harley quien insistió: "Colegas, que aquí el que se ha llevao hoy la pana he sío yo, que el mensaca jodío me ha dao plantón, me he quedao sin vender mi Maja [...]".

\footnotetext{
${ }^{8}$ En el siguiente enlace, el lector podrá comprobar la distribución entre España (50\% de los textos) y América $(50 \%)$ del CREA, y entre textos escritos $(90 \%)$ y orales $(10 \quad \%)$ : http://corpus.rae.es/ayuda_c.htm\#_Toc30228256

9 La Fundéu (https://www.fundeu.es/consulta/disyoquey-808/) propone adaptar este extranjerismo como disyóquey, pero no hemos hallado ninguna ocurrencia en los corpus de la RAE.
} 
Naveros, Miguel: Al calor del día. Madrid: Alfaguara, 2001. (CORPES XXI, consultado el 25/07/2017)

Citadas y justificadas las fuentes, vamos, a partir de ahora, a tratar de profundizar en las formas que nos atañen (véanse las tablas 1 y 2), principalmente desde la derivación por sufijación, la derivación apreciativa, que se halla en la frontera entre la morfología flexiva y la morfología léxica (Bergareche 1997; García Platero y Castillo Carballo 2006).

\begin{tabular}{|c|c|c|}
\hline & Préstamos & $\begin{array}{l}\text { chef } \mid \text { crack/crac } \mid \text { disc jockey (españolizado disyóquey, o DJ) - pinchadiscos } \\
\text { | líder } \mid \text { maître } \mid \text { sumiller | yóquey (jockey) }\end{array}$ \\
\hline \multirow{3}{*}{ 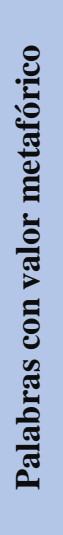 } & $\begin{array}{l}\text { Nombres proprios (de } \\
\text { N propio a } \mathbf{N} \text { común) }\end{array}$ & $\begin{array}{l}\text { (un) adán | (un) judas | (un) mecenas } \mid \text { (un) tarzán (una) mesalina } \mid \text { (una) } \\
\text { maruja }\end{array}$ \\
\hline & Nombres comunes & $\begin{array}{l}\text { (ser el) pilar (de...) } \mid \text { (ser un) ángel } \mid \text { (ser un) diamante (en bruto) } \\
\text { (ser un) engorro } \mid \text { (ser una) estrella (de) } \mid \text { (ser una) leyenda (viva) } \mid \\
\text { (ser un/a) máquina } \mid \text { (ser un/a) pelota } \mid \text { (ser una) perla } \mid \text { (ser un) plomo } \mid \text { (ser } \\
\text { una vieja) gloria }\end{array}$ \\
\hline & Nombres de animales & Cobaya | hormiguita | merluzo | pájaro, pajarraco | pichón | lince \\
\hline \multicolumn{2}{|c|}{$\begin{array}{l}\text { Voces terminadas en }-\mathbf{A} \text {, } \\
\text { procedentes de un neutron } \\
\text { griego }\end{array}$} & ácrata | demócrata $\mid$ jerarca $\mid$ hipócrita $\mid$ psicópata | patriarca \\
\hline \multirow{2}{*}{ 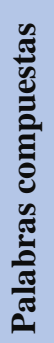 } & $\begin{array}{l}\text { Nombres } \\
\text { profesionales } \\
\text { ambas terminaciones o } \\
\text { invariables }\end{array}$ & cazatalentos $\mid$ picapleitos $\mid$ guardaespaldas $\mid$ pintamonas $\mid$ destripaterrones \\
\hline & Nombres calificadores & $\begin{array}{l}\text { cantamañanas } \mid \text { correveidile } \mid \text { mandamás* } \mid \text { marimacho* } \mid \text { metomentodo | } \\
\text { pelagatos } \mid \text { sabelotodo }\end{array}$ \\
\hline \multicolumn{2}{|c|}{$\begin{array}{c}\text { Adjetivos aparentemente } \\
\text { invariables }\end{array}$} & capaz $\mid$ cobarde $\mid$ cortés $\mid$ dócil $\mid$ hábil | histrión, -a | huésped, -a | tahúr \\
\hline \multicolumn{2}{|r|}{ Doble terminación } & $\begin{array}{l}\text { cateto, -a } \mid \text { cenizo, -a } \mid \text { enano, -a } \mid \text { matado, -a (matao/á) } \mid \text { monstruo, -a } \mid \\
\text { monstruito, -a } \mid \text { petardo, -a } \mid \text { tipo, -a }\end{array}$ \\
\hline
\end{tabular}

Tabla 4a: Clasificación de sustantivos según terminación

*En el caso de mandamás y marimacho, se documentan en femenino (mandamasa y marimacha). 


\begin{tabular}{|c|c|c|c|}
\hline \multirow{16}{*}{ 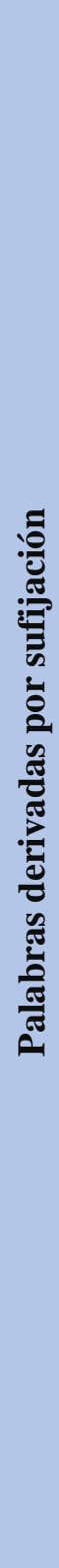 } & \multirow{9}{*}{ Forma terminadas en $-\mathrm{A}$} & $-\mathbf{a}$ & okupa/ocupa $\mid$ plasta $\mid$ trepa \\
\hline & & -aca & machaca $\mid$ sudaca \\
\hline & & -as & bocazas | pelanas \\
\hline & & -ata & drogata $\mid$ segurata $\mid$ sociata \\
\hline & & -era & hortera | lumbrera \\
\hline & & -ica & acusica | llorica \\
\hline & & -ista & arribista $\mid$ sablista \\
\hline & & $\begin{array}{l}- \text { ita(s) } \\
\text {-illa(s) }\end{array}$ & cocinillas $\mid$ cotilla $\mid$ manitas \\
\hline & & $\begin{array}{l}- \text { oca } \\
\text {-ota }\end{array}$ & carota $\mid$ masoca $\mid$ pasota \\
\hline & \multirow{4}{*}{ Forma terminadas en $-E$} & $-e$ & compinche | mequetrefe | zoquete \\
\hline & & $\begin{array}{l}\text {-ante } \\
\text {-ente }\end{array}$ & donante $\mid$ liante/-a $\mid$ maleante $\mid$ mangante $\mid$ traficante $\mid$ agente \\
\hline & & -ote & pasmarote \\
\hline & & -es & frescales | viejales | vivales \\
\hline & \multirow{2}{*}{ Forma terminadas en $-\mathrm{I}$} & $-\mathbf{i}$ & choni $\mid$ churri | friki $\mid$ mindundi | paganini | quinqui \\
\hline & & -is & finolis $\mid$ locatis $\mid$ millonetis \\
\hline & Forma terminadas en $-\mathrm{O}$ & $-\mathbf{0}$ & maromo $\mid$ tronco,-a \\
\hline
\end{tabular}

Tabla 4b: Clasificación de sustantivos según terminación

Inicialmente, agrupamos las formas que nos interesan por la vocal final. ¿Hay alguna razón? Para nosotras, sí la hay, y es una razón que nos parece de peso. Sea cual sea la vocal final, el hablante usará la forma indistintamente para referirse a una persona de género/sexo femenino o masculino. El uso del artículo será el indicador y, claro está, la estructura sintáctica en la que se organice la forma (el cotexto).

Luego, entre las formas terminadas en -a, hay varios sufijos (-aca: machaca; -ata: sociata, -oca/-ota: masoca, carota) que se han identificado como privativos de lenguajes sectoriales, ya sea por la edad de los locutores que las forman, ya sea por el ámbito aludido. También el conjunto de formas elegidas para la terminación en -i son propias de miembros de sectores marcados de la sociedad urbana actual. 
Hay otro grupo de formas que llevan inherente una marca de plural (-as: bocazas; -es: frescales; en -is: finolis o locatis; -itas: manitas; -illas: cocinillas).

Hay formas que presentan un sufijo de los llamados diminutivos o aumentativos (-ica: llorica; -illa(s): cocinillas, -ote: pasmarote).

Pues bien, en el uso de estas más o menos cuarenta formas nominales no hay ni rechazo de los finales en -e, en -i/-is, o en -o para aplicar a un referente de género/sexo femenino, ni al revés: las mayoría de las formas que terminan en -a no conocen transformación en -o para aplicarse a un referente de género/sexo masculino. Ya hemos hablado de maruja $\rightarrow$ marujo.

Si yo fuera presidenta, lo primero que haría sería instituir el día internacional del marujo para que dicho día las amas de casa fueran ellos y nosotras, repanchingarnos en el sofá. Bodega Estévez, Luisa... [et al.]: «Si yo fuera presidenta... Despedida y cierre». La maruja liberá. Madrid: Aguilar, 2005. [CORPES XXI, consultado el 25/07/2017]

Como en todos los casos comentados hasta ahora, la lengua elude toda ambigüedad con la aplicación del artículo, pero nos preguntamos: ¿siempre aparecerá un artículo junto a la forma?

El vocativo es una pieza esencial de la dinámica de la comunicación (Bañón 1993), sea como simple llamada, o como refuerzo, si el locutor acude a variantes léxico-semánticas en función apelativa. Como sabemos, el vocativo está cerca de la interjección y no forma parte del enunciado, por su papel metadiscursivo, pero los vocativos que aquí tratamos tienen un carácter referencial por aludir al receptor/oyente/lector/persona aludida.

El vocativo sirve para establecer, mantener, regular y consolidar la relación interpersonal. Con todo, las monografías consultadas (Bañón 1993; García Dini 1998; Gómez Montoya 2009) distinguen entre la pieza que sirve meramente para controlar el contacto, incluso físico, visual, de aquellos vocativos que constituyen una marca de afecto o de desafecto. Cuando el vocativo (caso de tío,-a, hombre/mujer; tronco, etc.) se usa para controlar el contacto, hay que considerarlo entre las partículas discursivas (Briz 2012; Jorgensen y Martínez 2009; Gelbes y Estrada 2003).

Hemos seleccionado, de la documentación allegada en los corpus, unos casos en los que es evidente a) el uso vocativo de la forma, b) la entonación exclamativa que confirma la apelación, y c) el resto de elementos que marcan o desambiguan cualquier posibilidad de confusión sobre el género/sexo del interlocutor aludido, incluso cuando la referencia es indirecta. Proponemos estos ejemplos ${ }^{10}$ :

¡Serás carota! Necesidad del contexto para saber si se refiere a masculino o a femenino, hombre o mujer.

iA la calle tía bruja, cotilla! Información cotextual (la forma termina en -a; también sabemos que es un calificativo aplicado con más frecuencia, por desgracia, a mujeres).

\footnotetext{
${ }^{10}$ Todos estos ejemplos proceden del CREA [consultado entre el 20 y el 30 de julio de 2017].
} 
¡Estáte quieta, pasmarote! Información cotextual (la forma termina en - ote; el adjetivo previo termina en -a).

¡Qué hombre, qué lumbrera! Información cotextual (la forma termina en -era) (Clarín, La Regenta).

-Habló el finolis (se sabe que quien habló antes era hombre).

“¿La finolis! ¡Lo ha plantado!” oyó decir a una muchacha (Juan Marsé, Ultimas tardes con Teresa).

Pelanas, eso es lo que me llamó (interpretación ambigua por la falta de artículo o demostrativo; es una referencia indirecta, además; reproducción de una intervención anterior).

En los resultados de la búsqueda en los corpus, se da esa presencia de artículos y aparecen tanto antes como después de la forma en cuestión:

- El artículo indeterminado precede a la forma: (ser) un/una jeta, un/una masoca, un compinche ${ }^{11}$, una liante, una manitas.

- El demostrativo precede a la forma (ese pelanas) o se pospone a ella (peyoración): el quinqui ese, la sudaca esa.

Gran parte de las veces, la ambigüedad se soluciona con el Npropio o una identificación: la bocazas de tu abuela, esa cantamañanas de Victoria, "tío Gonzalo era un muermo o un rollo o un plasta o un vara [...]”, ejemplo de un texto de A. Pombo. Cualquier variación ayuda: un plasta de hombre/una plasta de hombre/una plasta de mujer.

Mencionamos, de nuevo, la frecuencia de la estructura atributiva o, para ser más explícitas, de la función atributiva de ese nombre: ser un/a bocazas, que en posición de vocativo exclamativo puede darse sin el verbo: Sociata, el que no bote (no alude al voto, sino al saltito), parecer un cantamañanas, hecha un pasmarote, o de la función predicativa: actuar de machaca.

Llegamos a las conclusiones: nuestra revisión atenta de las 112 formas en su documentación de los corpus apoya la hipótesis de partida: fuera del ámbito de los nombres profesionales, en estas formas que han constituido en algún momento neologismos estilísticos (mediante la sufijación, mediante la aplicación metafórica), la tendencia a modificar la forma, marcando con una -a el género/sexo de la persona aludida no es sino parcial y se da en ambas direcciones (lianta, mandamasa, marimacha, marujo).

Lo atribuimos, en parte, a la gran variedad de sufijos apreciativos y a la heterogeneidad del sonido final del nombre resultante. Ocurre que el contenido semántico justifique la aplicación de una forma solo a hombres (tarzán, maromo -novio o amante-), o solo a mujeres (mesalina, marimacho -mujer que parece un hombre-). La información que puebla las redes sociales nos sirve para decir que choni, churri o guapi se dice de las chicas,

11 En nuestras búsquedas no hemos documentado ninguna ocurrencia referente a sujeto femenino (ni acompañada de una ni de la). 
que merluzo, baboso o cocinillas se dice de los hombres. Ahora bien, cuando las formas llegan al diccionario académico, el camino es más simple, dado que en la definición hallaremos mujer que..., hombre que..., o persona que...

Atribuimos la no alteración de la forma, también, al papel del artículo, que anula cualquier ambigüedad de interpretación. Una presencia a menudo redundada por otro determinante u otra referencia contextual. La atribuimos, además, al papel del cotexto y, sobre todo, a la situación comunicativa del enunciado.

Cabe incidir en el hecho de que se trata de formas apelativas que, con mayor frecuencia, conllevan connotación peyorativa (Rainer 2012a, 2012b). Quizá «peyoración» sea exagerado (sobre todo, vistos los trabajos de cortesía positiva y negativa), pero sí está claro que traducen una clara y personalizada actitud afectiva por parte de quien los usa (Beinhauer 1978, chap. III).

Estamos en una modalidad coloquial, pero no necesariamente exclusiva del discurso oral real. Dada la naturaleza de las fuentes aportadas por los corpus consultados, a menudo tales formas proceden de la prensa (de entrevistas, por ejemplo) o de textos de creación (sean teatrales o novelescos), en cuyo caso pertenecen a diálogos directos o a diálogos indirectos (reproducidos o evocados) y que, por tanto, pretenden reproducir el uso oral real de la lengua.

En definitiva, la afectividad es, en palabras de M. ${ }^{a}$ Lluïsa Hernanz (2001), «propiedad de índole semántico-pragmática asociada a determinadas piezas léxicas o construcciones, en virtud de las cuales estas adoptan un valor suplementario - ponderativo o evaluativo-que forma parte del contexto de interpretación».

\section{Bibliografía}

Alarcos Llorach, Emilio. 1999. Gramática de la lengua española. Madrid: Real Academia Española - Espasa Calpe.

Alcina, Juan y Bleuca José Manuel. 1975. Gramática española. Barcelona: Ariel, Editorial.

Bañón, Antonio Miguel. 1993. El vocativo: propuestas para su análisis lingüístico. Madrid: Ediciones Octaedro.

Beinhauer, Werner. 1978. El español coloquial. Madrid: Gredos.

Bello, Andrés. 1887. Gramática de la lengua castellana: destinada al uso de los americanos. Buenos Aires: Sopena.

Bergareche, Bruno Camus. 1997. "Sufijos apreciativos con derivados deverbales en español." Revista de Filología Románica 1 (14): 85-97.

Bosque, Ignacio. 1999. "El Nombre común." En Gramática descriptiva de la lengua española, 3-76. Madrid: Espasa.

. 2012. "Sexismo Lingüístico y visibilidad de la mujer." Real Academia de La Lengua Española - Informe.

Briz, Antonio. 2012. "La definición de las partículas discursivas 'hombre' y 'mujer"' Anuario de Lingüística Hispánica, Vol. 28, Valladolid: Universidad de Valladolid: 27-55. 
1990. "El proceso de sustantivación y lexicalización de los adjetivos con artículo en español.” Filología Románica 7 (231-239).

. 1998 [2001]. El español coloquial en la conversación: esbozo de pragmagramática. Barcelona: Ariel.

. 2010. "Lo coloquial y lo formal, el eje de la variedad lingüística."En De moneda nunca usada: estudios dedicados a José $M^{a}$ Enguita Utrilla, 2010. Zaragoza: Insituto Fernando El Católico, CSIC.

Cabré, M. Teresa, M. Rosa Bayà, Janet Ann de Cesaris y Elisenda Bernal. 2000. "Nombre propio y formación de palabras." En Gerd Wotjak (Coord.) En torno al sustantivo y adjetivo en el español actual: aspectos cognitivos, semánticos, (morfo)sintácticos y lexicogenéticos, 191-206. Madrid: Iberoamericana-Vervuert.

Chamizo, Pedro. 2005. La metáfora: semántica y pragmática. Recurso en línea, disponible en: https://www.ensayistas.org/critica/retorica/chamizo/.

Demonte, Violeta. 1999. "El adjetivo." En Gramática descriptiva de la lengua española, 129-216. Madrid: Espasa.

Fernández Ramírez, Salvador. 1985. Gramática Española. Madrid: Arco/Libros.

García, Aliaga, Fco, y Fernando Lázaro Mora. 2003. "La marcación de género en español." En Girón Alconchel, Girón Luis. Bustos Tovar, José Jesús de. (Eds.). Estudios ofrecidos al profesor José Jesús de Bustos Tovar, 5-22. Madrid: Editorial Complutense.

García Dini, Encarnación. 1998. "Algo más sobre el vocativo.” Sogno e scrittura nelle culture iberiche: Atti del XVII Convegno: Milano, 24-26 Ottobre 1996. Bulzoni, 57-62.

García Platero, Juan Manuel y María Auxiliadora Castillo Carballo. 2006. "Valor pragmático de la apreciación en español." En La Competencia pragmática y la enseñanza del español como lengua extranjera, 2006, 208-12. Oviedo: Ediciones de la Universidad de Oviedo, Servicio de Publicaciones.

Gelbes, S. R., y A. Estrada. 2003. "Vocativos insultivos vs. vocativos insultativos: Acerca del caso de 'boludo'." Anuario de Estudios Filológicos: 335-353, 26. Extremadura: Universidad de Extremadura. Facultad de Filosofía y Letras.

Gómez Montoya, John Jairo. 2009. "El vocativo en la interacción escritor-lector." Mutatis Mutandis: Revista Latinoamericana de Traducción, (Ejemplar dedicado a: pedagogía y didáctica de la traducción II), 2 (2). Colombia: Universidad de Antioquia: 313-26.

Gómez Torrego, Leonardo. 2011. "Reflejo de algunos cambios sociales en el español actual." Revista Cálamo, no. 58. FASPE: 8-24.

González Rodríguez, Raquel. 2009. "Algunas observaciones sobre la distribución de los elativos en español.” LEA: Lingüística Española Actual, 31 (2): 221-41.

Hellinger, Marlis, y Hadumod Bußmann. 2002. Gender across Languages: The Linguistic Representation of Women and Men. Vol. 2. J. Benjamins.

Hernández Alonso, César. 1991. "El lenguaje coloquial juvenil.” Revista de La Asociación Europea de Profesores de Español, no. 38-39. Instituto Cervantes.

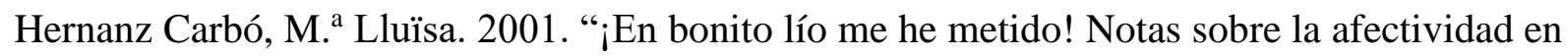
español." Moenia: Revista Lucense de Lingüistica y Literatura, no. 7. Santiago de Compostela: Universidade de Santiago de Compostela, Servicio de Publicacións e Intercambio Científico: 93-109. 
Jorgensen, Annette Myre y Juan Antonio Martínez. 2009. “Tronco/a' usado como marcador discursivo en el lenguaje juvenil de Madrid.". Disponible en: https://www.researchgate.net/publication/215958619_'Troncoa'_usado_como_marcad or_discursivo_en_el_lenguaje_juvenil_de_Madrid

Junyent, M. Carme. 2013a. "La variable 'sexe' en les llengües del món”. Caplletra 54: 14358.

Junyent, M. Carme. 2013b. Visibilitzar o marcar: repensar el gènere en la llengua catalana. Barcelona, Empúries.

Larsson, Christel. 2008. "La flexión de género femenino en los nombres de oficios, cargos y profesiones en el español peninsular contemporáneo.” Goteborgs Universitet.

- 2014. ¿La fiscal, la fiscala o la mujer fiscal? Un estudio sociolingüístico sobre el nombramiento de mujeres trabajadoras, Göteborgs universitet, Opuscula Romanica.

Llamas Saíz, Carmen. 2005. Metáfora y creación léxica. Pamplona: Ediciones Universidad de Navarra.

Lledó Cunill, Eulàlia. 2011. Manual de llengua per visibilitzar la presència femenina. Barcelona: Unió de Federacions Esportives de Catalunya - BEAT. Recurso en línea, disponible en: http://www.beat.cat/manualdellengua/.

Manrique Sabogal, Winston. 2012. “La lengua tiene género? ¿Y sexo?” Cultura|EL PAÍS, March 5. Disponible en:

https://elpais.com/cultura/2012/03/04/actualidad/1330896843_065369.html

Marimón-Llorca, Carmen y Isabel Santamaría-Pérez. 2001. "Procedimientos de creación léxica en el lenguaje juvenil universitario." Estudios de Lingüística. 15. Alicante: Universidad de Alicante, Departamento de Lengua Española, Facultad de Filosofía y Letras: $87-114$.

Martinell, Emma, 1973. Encadenamiento por repetición en la estructura coloquial. Tesis doctoral, Universitat de Barcelona Facultat de Filosofia i Lletres. Disponible en: http://www.tdx.cat/handle/10803/1712

Moral, Rafael del. 2003. Manual práctico del español coloquial: con 50 ilustraciones. Madrid: Editorial Verbum.

Rainer, Franz. 2012a. "El patrón industria manufacturera la influencia francesa en el uso 'económico' de los adjetivos en -ero." En Assí como es de suso dicho: estudios de morfología y léxico en homenaje a Jesús Pena, 453-68. San Millán de la Cogolla: Cilengua (Grupo USC FILGA).

2012b. "Escarceos sobre la conversión sustantivo adjetivo en español." In Los límites de la morfología: estudios ofrecidos a Soledad Varela Ortega, Barcelona: Universitat Autònoma de Barcelona: 369-82.

Real Academia Española. 1973. Esbozo de una nueva gramática de la lengua española. Madrid: Espasa-Calpe 2010. Nueva gramática de la lengua española. Madrid: Espasa-Calpe.

Rodríguez González, Félix. 2002. "Lenguaje y contracultura juvenil: anatomía de una generación.” En El lenguaje de los jóvenes, 29-56. Barcelona: Ariel.

. 2002. El lenguaje de los jóvenes. Barcelona: Ariel. 
Serrano-Dolader, David. 2010. “El género en los sustantivos: ¿flexión y/o derivación?” José Francisco Val Álvaro y M. ${ }^{\mathrm{a}}$ Del Carmen Horno Chéliz (Eds.): La gramática del sentido: léxico y sintaxis en la encrucijada, [Colección Knowledge-LanguageCommunication / Conocimiento-Lenguaje-Comunicación] 3: 249-70.

Timofeeva, Larissa. 2005. "La ironía en las unidades fraseológicas." Interlingüística (ejemplar dedicado a: XX Encuentro de La Asociación de Jóvenes Lingüistas), Págs. 1069-1077, no. 16. Barcelona: Universitat Autònoma de Barcelona: 1069-77.

Vigara Tauste, Ana María. 1992. Morfosintaxis del español coloquial: Esbozo estilístico. Madrid: Editorial Gredos.

Zimmermann, Klaus. 2002. "La variedad juvenil y la interacción verbal entre jóvenes.” En Thomas Kotschi, Wulf Oesterreicher y Klaus Zimmermann (eds.), El español hablado y la cultura oral en España e Hispanoamérica, Madrid-Frankfurt a.M.: Iberoamericana-Vervuert: 476-514. 